\title{
Arbeitsmarktreformen in Deutschland: Eine noch unvollendete Erfolgsgeschichte
}

Der deutsche Arbeitsmarkt hat in den letzten Jahren in vielfacher Hinsicht positiv überrascht, vor allem angesichts der desolaten Ausgangslage in den Vorjahren: Im jüngsten Aufschwung zeigen sich eine markante Beschäftigungszunahme sowie Tiefstände bei der Arbeitslosigkeit; in der schwersten Rezession nach dem Krieg bewies er eine ungeahnte Widerstandsfähigkeit. Lassen sich diese Entwicklungen mit den vorangehenden Arbeitsmarkt- und Sozialreformen erklären, den wohl umfassendsten der letzten Jahrzehnte? Der Beitrag antwortet mit einem „Ja, aber“: Dank der Reformen sind der deutsche Arbeitsmarkt und die deutsche Volkswirtschaft in einer deutlich besseren Verfassung. Doch nicht alle Probleme konnten mit den bisherigen Maßnahmen gelöst werden.

\section{Ausgangslage: Zentrale Probleme auf dem Arbeitsmarkt}

Zu Beginn der letzten Dekade wies der deutsche Arbeitsmarkt eine Reihe gravierender Probleme auf. Für sich genommen waren sie nicht neu, hatten sich aber über die Zeit hinweg verstärkt und in einem ungünstigen makroökonomischen Umfeld derart zugespitzt, dass nun die Bereitschaft zu umfassenden Reformmaßnahmen bestand. $\mathrm{Zu}$ diesen Problemen zählten insbesondere

- eine hohe und mit jedem Zyklus weiter steigende strukturelle Arbeitslosigkeit;

- hohe Arbeitslosigkeit unter Geringqualifizierten und ein hoher Anteil an Langzeitarbeitslosen. Beide Probleme waren auch im internationalen Vergleich außergewöhnlich hoch;

- eine niedrige Erwerbsbeteiligung Älterer mit hohen Kosten für den Fiskus und negativen Folgen für das Potenzialwachstum, teilweise befördert durch teure, die Arbeitslosenversicherung und die Gesetzliche Rentenversicherung belastende Programme, die auf das frühzeitige Ausscheiden aus dem Erwerbsleben zielten (wie Altersteilzeit, „58er-Regel“ oder vorzeitige Rente wegen Arbeitslosigkeit);

- anhaltende Defizite bei der Bundesanstalt für Arbeit trotz eines hohen Beitragssatzes, verbunden mit der vermuteten oder nachgewiesenen Ineffizienz bedeutender
Ausgabeposten und fehlendem Wissen über die tatsächliche Wirksamkeit wichtiger Maßnahmen. Die Folge war, dass die Beiträge zur Arbeitslosenversicherung nur noch begrenzten Äquivalenzcharakter hatten - die einkommensabhängigen Beiträge überstiegen merklich den Wert der damit verbundenen einkommensabhängigen Leistungsansprüche - und somit einen beschäftigungsfeindlichen hohen Steueranteil aufwiesen (SVR 2005, Tz. 507ff.);

- die anhaltende Spaltung des Arbeitsmarktes zwischen Ost- und Westdeutschland;

- eine rückläufige Bedeutung sozialversicherungspflichtiger Beschäftigungsverhältnisse und damit der Einnahmebasis für die Sozialversicherungen.

Neben diesen als drängend erkannten Problemen auf dem Arbeitsmarkt begünstigten im Jahr 2002 auch politökonomische Gründe umfassendere Reformen - insbesondere anstehende Wahlen und ein öffentlicher Meinungsumschwung im Zuge von Unregelmäßigkeiten bei der Vermittlungsstatistik der damaligen Bundesanstalt für Arbeit (SVR 2002, Tz. 188ff.).

\section{2 \\ Reformmaßnahmen und Reformziele}

Reformen am Arbeitsmarkt hatte es bereits vor dem Jahr 2002 gegeben. Die durch die
Hartz-Kommission und insbesondere die Agenda 2010 angestoßenen Maßnahmen hatten indes eine neue Qualität und veränderten die Verfasstheit des deutschen Arbeitsmarktes sowie die sozialen Grundsicherungssysteme („Sozialhilfe“) nachhaltig. Mit Blick auf die Arbeitsmarktwirkung der Reformen und die Bekämpfung der genannten Probleme ist es wichtig, diese Reformen umfassend und nicht begrenzt auf die „Hartz-Gesetze“ zu verstehen. Hauptansatzpunkte waren:

(1) Die Lohnersatzleistungen: Neben der Verkürzung der Bezugsdauer des Arbeitslosengelds ging es hier vor allem um die $\mathrm{Zu}$ sammenlegung von Arbeitslosenhilfe und Sozialhilfe zur Grundsicherung für Arbeitsuchende als einheitlicher bedürftigkeitsabhängiger Leistung für Erwerbsfähige. Für die vormaligen Sozialhilfeempfänger bedeutete dies eine Besserstellung beim Transfervolumen, vor allem aber beim Zugang zu Leistungen der Arbeitsmarktförderung (Konle-Seidl et al. 2007).

\footnotetext{
Stephan Kohns, Dr., ist Mitarbeiter der Deutschen Bundesbank und gehörte von 2002 bis 2008 dem wissenschaftlichen Stab des Sachverständigenrates zur Begutachtung der gesamtwirtschaftlichen Entwicklung (SVR) an, darunter von 2005 bis 2008 als Generalsekretär. Der Autor vertritt hier seine persönliche Auffassung. e-mail: stephan.kohns@gmx.de
} 
(2) Die aktive Arbeitsmarktpolitik: Sie sollte mittels einer Durchforstung des bisherigen Instrumentariums sowie der Einführung neuer Instrumente auf eine stärkere Aktivierung und eine passgenauere Förderung von Arbeitsuchenden ausgerichtet werden.

(3) Das Mandat und die Organisation der Arbeitsmarktverwaltung: Sichtbarster Ausdruck des Neuordnungsprozesses waren hier die Umbenennung der Arbeitsämter in Agenturen für Arbeit sowie die verstärkte Zusammenarbeit von Agenturen und Kommunen bei der Grundsicherung für Arbeitsuchende.

(4) Die Modalitäten des Ausscheidens aus dem Erwerbsleben: Neben der schrittweisen Rücknahme von Anreizen zur Frühverrentung ging es auch um die Rentenreform und die Ankündigung einer schrittweisen Anhebung des gesetzlichen Renteneintrittsalters.

(5) Arbeits- und sozialrechtliche Lockerungen bei den atypischen Beschäftigungsverhältnissen (geringfügige Beschäftigung, befristete Beschäftigung und Leiharbeit): Diese Maßnahmen zielten darauf, sowohl den Einstieg in den ersten Arbeitsmarkt als auch die Anpassung der unternehmerischen Arbeitsnachfrage an vorübergehenden Mehr- oder Minderbedarf zu erleichtern. Da der Kündigungsschutz im Wesentlichen unangetastet blieb, fand im Ergebnis eine „Flexibilisierung an den Rändern“ statt, womit die bereits bestehende Segmentierung des Arbeitsmarktes in Kernbelegschaften mit hohem Schutzniveau und Rand- oder Zusatzbeschäftigte, von denen eine höhere Anpassungsbereitschaft erwartet wird, noch vertieft wurde.

Diese Maßnahmen zielen in unterschiedlicher Weise auf die einzelnen vorgenannten Probleme, wobei die intendierten Wirkungen häufig mittelbar und nicht immer gleichgerichtet sind.

\section{Evidenz zur Bewährung der Reformen}

Die durch die Reformen anzugehenden Probleme waren ganz überwiegend struktureller Natur, was die Bewertung der Reformen anhand lediglich einer abgeschlos- senen Aufschwungphase (2005-2008) und eines mittlerweile abgeschlossenen Abschwungs (2008-2009), die zudem beide auf ihre Art außergewöhnlich waren, erschwert. Hinzu kommen arbeitsmarktstatistische Probleme, etwa Umstellungen in der Abgrenzung der registrierten Arbeitslosigkeit (SVR 2005, Anhang B Tz. 3). Obwohl sich ein abschließendes Urteil noch verbietet und eine Überprüfung teilweise eklektisch vorgehen muss, ist eine vorläufige Bestandsaufnahme gleichwohl möglich.

\subsection{ARBEITSMARKTGESCHEHEN VOR UND NACH DEN REFORMEN}

Um einzuschätzen, ob die Arbeitsmarktentwicklung seit Umsetzung der Reformen Indizien für die Wirkung der Reformen liefert, bietet sich zunächst ein Vergleich mit früheren Jahren an, etwa über einen expliziten Zyklenvergleich, wie er verschiedentlich vorgenommen wurde (SVR 2007, Tz. 481ff.; SVR 2008, Tz. 475ff.; Horn et al. 2007; Horn et al. 2008). Im Folgenden wird dazu die vom Sachverständigenrat zur Begutachtung der gesamtwirtschaftlichen Entwicklung (SVR) verwendete Abgrenzung der Konjunkturzyklen zugrunde gelegt, der zufolge ein Aufschwung vom Tiefpunkt des gesamtwirtschaftlichen Auslastungsgrades bis zum nachfolgenden Hochpunkt reicht. ${ }^{1}$ Gemäß diesem Ansatz begann der jüngste und mit Abstand längste Aufschwung im Schlussquartal 2004, der vorangegangene und - gemessen an der Entwicklung des Bruttoinlandsprodukts (BIP) etwas dynamischere - im 2. Quartal 1999. Ergänzend können zur Wirkungsabschätzung um den Konjunktureinfluss bereinigte Größen oder die Entwicklung in anderen Volkswirtschaften herangezogen werden.

Die Entwicklung der Arbeitslosigkeit, die ja einen der maßgeblichen Reformanstöße gegeben hat, hebt sich seit dem Jahr 2005 deutlich von früheren Mustern ab. So gelang es im vergangenen Aufschwung erstmals, den Sperrklinkeneffekt zu durchbrechen, dem zufolge in jedem neuen Konjunkturzyklus der Tiefpunkt der Arbeitslosenquote höher lag als in den vorangehenden Zyklen. Dieser Rückgang ist umso bemerkenswerter, als es im Zuge der Einführung der Grundsicherung zu einem erfassungsbedingten Anstieg der Arbeitslosigkeit kam. Das aber heißt: Die zu beobachtende Entwicklung deutet auf einen Rückgang der strukturellen Ar- beitslosigkeit hin. Dafür sprechen ebenso direkte Schätzungen der strukturellen (inflationsstabilen) Arbeitslosenquote wie das Verhältnis von Arbeitslosigkeit und offenen Stellen, die sogenannte Beveridgekurve (SVR 2008, Tz. 477; Möller et al. 2009), deren Rückverlagerung auf ein besseres Zusammenfinden von offenen Stellen und Arbeitsuchenden hindeutet. Mittelbar lässt zudem die trotz der dynamischen Konjunktur verhaltene Lohnentwicklung, also das Fehlen von Lohndruck bis hin zu sinkenden Reallöhnen bei gleichzeitig sinkender Arbeitslosigkeit, auf eine rückläufige strukturelle Arbeitslosigkeit schließen: Die für sich genommen lohnerhöhend wirkende steigende Nachfrage nach Arbeitskräften wurde durch eine stärkere Mobilisierung von bislang Arbeitslosen ausgeglichen oder sogar überkompensiert.

Bei der Arbeitsmarktlage der besonderen Problemgruppen aufdem Arbeitsmarkt ist das Bild weniger eindeutig: Im Bereich der Grundsicherung für Arbeitsuchende hat die Arbeitslosigkeit ebenfalls abgenommen. In dieser Gruppe finden sich in der Tat besonders viele Geringqualifizierte und Langzeitarbeitslose - so waren im Jahr 2005, als die Grundsicherung eingeführt wurde, drei Viertel der entsprechenden Leistungsbezieher gering qualifiziert oder langzeitarbeitslos, und drei Viertel aller Arbeitslosen, die gering qualifiziert oder langzeitarbeitslos waren, bezogen Leistungen nach dem SGB II (SVR 2006a, Tabelle 4). Allerdings erfolgte der Rückgang der Arbeitslosigkeit im SGB-II-Bereich später und nicht so stark wie im Bereich des SGB III, der typischerweise die Bezieher von Arbeitslosengeld erfasst. Die Langzeitarbeitslosigkeit insgesamt ging zwar stärker als in der Vergangenheit zurück, aber der Rückgang ist auch der aktiven Arbeitsmarktpolitik geschuldet, während sich die Übergänge in den ersten Arbeitsmarkt verglichen mit dem vorangehenden Aufschwung nur etwas erhöht haben (SVR 2009, Tz. 467ff.). Im internationalen Vergleich sind die Langzeitarbeitslosenquote und der Anteil der Langzeitarbeitslosen an allen Arbeitslosen weiterhin ungewöhnlich hoch, und auch die Arbeitslosenquote der Geringqualifizierten liegt noch deutlich

1 Siehe SVR (2007, Kasten 14). Horn et al. (2008) datieren den Beginn des zweiten Aufschwungs ein Jahr früher und kommen dadurch zu einer etwas anderen Einschätzung der relativen Stärke der Aufschwungphasen. 
über dem internationalen Durchschnitt. Positiv zu vermerken ist immerhin, dass es in Ostdeutschland ebenfalls zu einem deutlichen Rückgang der Arbeitslosigkeit gekommen ist und dass die Arbeitslosigkeit unter Älteren überdurchschnittlich stark abgenommen hat (Arlt et al. 2009), und zwar trotz Rücknahme der Förderung eines vorzeitigen Ausscheidens aus dem Erwerbsleben.

Die Erwerbstätigkeit nahm im jüngsten Aufschwung besonders stark zu und erreichte im Jahr 2008 mit 40,3 Mio. Personen einen neuen Höchststand. Nun mag man einwenden, dass dies vor allem der längeren Dauer des Aufschwungs geschuldet sei (vgl. Sturn/van Treeck in diesem Heft). Dem ist zweierlei entgegenzuhalten: Zum einen nahm im jüngsten Aufschwung, anders als im vorangehenden, die am Erwerbspersonenpotenzial gemessene Zahl der verfügbaren Arbeitskräfte nicht mehr zu. Zum anderen hatte der Anstieg der Erwerbstätigkeit in mehrfacher Hinsicht eine andere Qualität. Beispielsweise verliefen im jüngsten Aufschwung Erwerbstätigenzahl und gesamtes Arbeitsvolumen weitgehend symmetrisch, während im Aufschwung der Jahre 1999 bis 2001 das Arbeitsvolumen nach einigen Quartalen stagniert hatte. Darin kommt zum Ausdruck, dass - verglichen mit früheren Zyklen - im letzten Aufschwung die sozialversicherungspflichtige Beschäftigung und die Zahl der Vollzeitstellen merklich zunahmen, während sich gerade in der vorangehenden Boomphase der Anstieg der Erwerbstätigkeit stärker auf geringfügige Beschäftigungsverhältnisse und andere Teilzeitformen gestützt hatte, mithin auf Arbeitsverhältnisse, die weniger zum Arbeitsvolumen beitragen. Insgesamt konnte bis 2008 der seit 2001 beobachtbare markante Rückgang bei der Zahl der sozialversicherungspflichtig Beschäftigten wieder wettgemacht werden: in Deutschland insgesamt immerhin zu einem beträchtlichen Teil und in Westdeutschland sogar gänzlich.

Die überaus lebhafte Beschäftigungsentwicklung lässt sich auch nicht mit dem Verweis auf den rapiden Anstieg der Arbeitnehmerüberlassung als Teil der sozialversicherungspflichtigen Beschäftigung abtun. Zwar nahm im vergangenen Aufschwung die Zahl der Leiharbeitnehmer in der Tat kräftig von rund 400.000 im Schlussquartal 2004 auf über $700.000 \mathrm{im}$ 1. Quartal 2008 zu. Doch erstens belief sich der Anstieg der sozialversicherungspflichtigen Beschäftigung im entsprechenden Zeitraum auf deutlich über 800.000 Personen, kann also nur zu knapp zwei Fünfteln mit der Leiharbeit erklärt werden. Zweitens sind Leiharbeitsverhältnisse zwar weniger stabil und somit für die Betroffenen meist weniger attraktiv, doch handelt es sich bei ihnen genauso um reguläre, nicht aus öffentlichen Mitteln geförderte Beschäftigungsverhältnisse auf dem ersten Arbeitsmarkt mit einem genuinen Beitrag zur gesamtwirtschaftlichen Wertschöpfung wie ihn auch die übrigen Arbeitnehmer in den Entleihbetrieben erbringen. Anders als beispielsweise Beschäftigungsverhältnisse auf dem zweiten Arbeitsmarkt ist schon deshalb das Segment der Arbeitnehmerüberlassung kein geeigneter Beleg, um die Beschäftigungsentwicklung im Aufschwung zu relativieren.

Eine außergewöhnliche Arbeitsmarktdynamik im jüngsten Aufschwung wird ferner durch einen internationalen Vergleich der Erwerbsbeteiligung belegt, die zwischen den Jahren 2005 und 2008 in Deutschland deutlich stärker zunahm als etwa in der EU-15 oder der gesamten OECD. Dies gilt sogar noch stärker für die Gruppe der 55- bis 64-Jährigen: Hier verzeichnete die Erwerbsbeteiligung in Deutschland nach der Slowakei und gleichauf mit den Niederlanden den in Prozentpunkten gemessenen stärksten Anstieg unter den OECD-Ländern (OECD 2009).

In der Summe zeigt sich somit, dass sich die Entwicklung von Arbeitslosigkeit, Erwerbstätigkeit und Erwerbsbeteiligung nach Umsetzung der Reformen spürbar und vor allem positiv von früheren Jahren abhebt. Die erzielten Fortschritte bei der Bekämpfung der eingangs genannten Probleme liefern zwar keinen formalen Beweis für einen kausalen Zusammenhang mit den Reformen, wohl aber ein starkes Indiz.

\subsection{ARBEITSLOSENVERSICHERUNG: HAUSHALTSLAGE UND HAUSHALTS- STRUKTUR}

Der augenfälligste Reformerfolg vor der Rezession ist die dramatische Verbesserung der Haushaltslage in der Arbeitslosenversicherung. Zwar wurde die Arbeitslosenversicherung zulasten des Bundeshaushalts von Ausgaben befreit, indem die Finanzierung der aktiven Arbeitsmarktpolitik für Langzeitarbeitslose von Beitrags- weitgehend auf Steuermittel umgestellt und die maximale Bezugsdauer des Arbeitslosengelds verkürzt wurde. Den Umschwung in der Finanzlage der Arbeitslosenversicherung haben indes vor allem die gute Beschäftigungsentwicklung, deutlich rückläufige Arbeitslosenzahlen insbesondere im Rechtskreis des SGB III und eine massive Umgestaltung der aktiven Arbeitsmarktpolitik bewirkt. Trotz sukzessiver Beitragssatzsenkungen von ursprünglich 6,5 auf 2,8\% konnten beträchtliche Überschüsse erzielt und auf diese Weise Rücklagen in zweistelliger Milliardenhöhe aufgebaut werden, die zumindest in der ersten Phase der Wirtschaftskrise die Verschlechterung der Finanzlage abfederten. Obwohl die Arbeitslosenversicherung mit dem aktuellen Beitragssatzniveau wieder unterfinanziert ist (SVR 2007, Tz. 316ff.), fiel somit eine beträchtliche finanzpolitische Reformdividende an.

Noch wichtiger als diese buchhalterische Verbesserung der Haushaltslage sind indes die qualitativen Veränderungen und das erkennbare Bestreben, den Instrumenteneinsatz noch stärker am Erfolg der Reintegration in den Arbeitsmarkt auszurichten. Eine wichtige Rolle spielte dabei die umfassende, wissenschaftlich begleitete Evaluation bestehender und neuer arbeitsmarktpolitischer Instrumente, die schon für sich genommen einen wichtigen, reformbedingten Fortschritt darstellt. Denn eine sorgfältige, auf den kausalen Effekt von Maßnahmen zielende Wirkungsanalyse, die auch Einsperr-, Mitnahme-, Selektions- und Verdrängungseffekte explizit mit berücksichtigt, ist eine Grundvoraussetzung für einen effizienten Mitteleinsatz (SVR 2003, Tz. 241). Darüber hinaus wird der politische Wille benötigt, die Evaluationsbefunde auch umzusetzen. Dass es daran bisweilen noch mangelt, zeigt das Beispiel von Überbrückungsgeld und Ich-AG, die trotz jeweils günstiger Evaluationsergebnisse abgeschafft und zu einem Instrument zusammengelegt wurden (SVR 2006b, Tz. 507).

Angesichts der Kürze der seit der Einführung vieler neuer Maßnahmen verstrichenen Zeit sind kaum Aussagen über Langfristfolgen möglich. Und auch zur aktuell wichtigen Frage der Wirkung der aktiven Arbeitsmarkpolitik im Konjunkturzyklus gibt es bislang kaum Evidenz. Die vorhandenen Studien deuten aber darauf hin, dass sich die Wirksamkeit des Mitteleinsatzes erhöht hat und, wie auch schon aus anderen Ländern bekannt, 
aktivierende Maßnahmen mit einer besonderen Nähe zum ersten Arbeitsmarkt, wie z. B. Existenzgründungshilfen, zielgerichtete Lohnzuschüsse oder kurzzeitige, fokussierte Qualifizierungsmaßnahmen, Erfolg versprechend sind (Caliendo 2009; Jacobi/Kluve 2007; Bernhard et al. 2009). Andere Instrumente haben demgegenüber nur einen geringen positiven oder unter Umständen sogar einen negativen Effekt auf die Arbeitsmarktchancen der Teilnehmer. Von der geringfügigen Beschäftigung scheinen in der Summe zwar keine Verdrängungseffekte auf reguläre Beschäftigung auszugehen, die Erwartung einer Brücke in den ersten Arbeitsmarkt hat sich aber nicht erfüllt. Hervorzuheben ist auch, dass im Einklang mit empirischen Studien für andere Länder die Verkürzung der Bezugsdauer die Eintritte in die Arbeitslosigkeit merklich gesenkt und somit die Arbeitslosenversicherung nicht nur buchhalterisch, sondern auch durch Verhaltensänderungen der Leistungsempfänger infolge der kürzeren Bezugsdauer entlastet hat (Dlugosz et al. 2009). Umso bedauerlicher ist, dass dieses Reformelement Anfang 2008 teilweise wieder zurückgenommen wurde - die maximale Bezugsdauer für Arbeitslosengeld beläuft sich nunmehr auf zwei Jahre.

\subsection{DER DEUTSCHE ARBEITSMARKT IN DER FINANZ- UND WIRTSCHAFTS- KRISE}

Die erneute Zuspitzung der Finanzkrise im Herbst 2008 und die sich zu diesem Zeitpunkt ohnehin bereits abkühlende Weltkonjunktur stürzten die Weltwirtschaft und vor allem die entwickelten Volkswirtschaften in die tiefste Rezession der Nachkriegszeit. Die Wirtschaftsleistung brach im Winterhalbjahr 2008/2009 massiv ein. Und obgleich die Abwärtsdynamik durch massive Stützungsmaßnahmen von Geld- und Fiskalpolitik gestoppt wurde und ab dem Frühjahr 2009 eine Stabilisierung eintrat, ging das deutsche Bruttoinlandsprodukt im Jahr 2009 um $4,7 \%$ zurück. Ex ante hätte es nahegelegen $\mathrm{zu}$ vermuten, dass sich bei einer derartigen Rezession die reformbedingt höhere Flexibilität auf dem Arbeitsmarkt in einem rascheren Abbau der Beschäftigung, zumindest bei den als Nachfragepuffer eingesetzten atypischen Formen, und einem Anstieg der Arbeitslosigkeit äußert. Stattdessen nahm die Erwerbstätigkeit noch bis in den Herbst 2009 zu, und die
Arbeitslosigkeit stieg nur sehr verhalten an. Zwar ist die Zunahme der registrierten Arbeitslosigkeit zugegebenermaßen arbeitsmarktstatistisch etwas unterzeichnet (BA 2010, S. 27), aber auch die Stromgrößen, d.h. Zugänge in und Abgänge aus Arbeitslosigkeit, zeigen keinen rezessionsbedingten Bruch (vgl. Rosemann/Kirchmann in diesem Heft). Im Einklang damit blieb die Einstellungsbereitschaft der Unternehmen robust, wenn man beispielsweise den von der Bundesagentur für Arbeit berechneten Index BA-X zugrunde legt, der die Anzahl der offenen Stellen ausweist (Bundesbank 2010a). Während lange erwartet wurde, ein stärkeres Durchschlagen der Krise auf den Arbeitsmarkt sei nur aufgeschoben, gehen aktuelle Prognosen inzwischen davon aus, dass es 2010 zu keinem Einbruch der Beschäftigung mehr kommt. Vielmehr dürfte die Talsohle auf dem Arbeitsmarkt inzwischen durchschritten sein.

Diese überraschende Entwicklung ist mehreren Faktoren geschuldet und nicht allein auf die vergangenen Reformen zurückzuführen. Zerlegt man die Entwicklung der Erwerbstätigkeit nach Branchen, so hatte das exportabhängige und daher von der Krise besonders getroffene Verarbeitende Gewerbe durchaus merkliche Beschäftigungsverluste zu verzeichnen, die aber durch einen anhaltenden Beschäftigungsaufbau im Dienstleistungsbereich mehr als kompensiert wurden (Bundesbank 2009). Diese Konzentration des Beschäftigungsabbaus auf hochproduktive Branchen bedeutet auch, dass die traditionellen, eher dem Rechtskreis SGB II zuzuordnenden Problemgruppen des Arbeitsmarktes von der Krise bisher eher verschont geblieben sind (Möller et al. 2009). Gleiches gilt für die befristet Beschäftigten, deren Anteil anders als der der Leiharbeitnehmer in den exportorientierten Branchen unterdurchschnittlich ist (Hohendanner 2010). Bei der stärker in exportabhängigen Branchen vertretenen Leiharbeit kam es in der Tat zu einem deutlichen Einbruch der Beschäftigtenzahl, die inzwischen aber wieder stetig zunimmt.

Das Arbeitsvolumen hat sich mit einem Rückgang von 2,8 \% im Jahr 2009 bei Weitem nicht so stark verringert wie das Bruttoinlandsprodukt, und so ist die Stundenproduktivität deutlich gesunken. Gleichwohl hat die durchschnittliche Arbeitszeit abgenommen. Eine weitere Aufspaltung des Rückgangs der Arbeitszeit zeigt, dass dieser zu etwa einem Drittel auf die Nutzung von Kurzarbeit, zum größeren Teil aber auf den Abbau von Arbeitszeitkonten und Überstunden, eine Verringerung der regulären Arbeitszeit und einen höheren Anteil von Teilzeitarbeit zurückzuführen ist (vgl. Herzog-Stein/ Seifert in diesem Heft). Offenkundig hat die Nutzung unterschiedlicher Formen der innerbetrieblichen Flexibilität bei der Vermeidung von Entlassungen eine ganz entscheidende Rolle gespielt. Dieses Verhalten dürfte zum einen auf eine reformbedingt größere Konzessionsbereitschaft von Arbeitnehmern, Betriebsräten und Gewerkschaften zurückzuführen sein, etwa weil Beschäftigte im Falle der Entlassung nun schneller auf die bedürftigkeitsabhängige Grundsicherung angewiesen wären. Im Einklang mit einer derartigen arbeitsangebotsseitigen Erklärung steht, dass in den bisherigen Tarifrunden die Sicherung der Beschäftigung gegenüber Einkommenszuwächsen klaren Vorrang hatte. Nicht von der Hand zu weisen sind zum anderen aber arbeitsnachfrageseitige Erklärungen, d.h. das Bestreben der Unternehmen, ihre Arbeitskräfte möglichst zu halten. Möglicherweise tritt hier der besondere Charakter der Krise zutage, die zunächst vor allem Branchen traf, die durch ein hohes firmenspezifisches Wissen der Arbeitnehmer, die Sorge um die Deckung des künftigen Fachkräftebedarfs und eine ohnehin höhere Volatilität der Kundennachfrage im Konjunkturzyklus charakterisiert sind. Alle diese Faktoren begünstigen auch aus Arbeitgebersicht die Hortung von Arbeitskräften, sofern die Krise und die Unterauslastung der Kapazitäten als vorübergehend und überbrückbar erscheinen.

Festzuhalten bleibt damit, dass der deutsche Arbeitsmarkt in der Krise in der Tat eine ungewöhnliche Widerstandsfähigkeit gezeigt hat. Dies dürfte zum Teil auf den besonderen Charakter der Krise und die Stärke des vorangehenden Aufschwungs zurückzuführen sein; zum anderen aber auch auf eine veränderte Dynamik: sowohl des Arbeitsmarktgeschehens insgesamt als auch bei den Entscheidungen über Löhne und Beschäftigung in den einzelnen Betrieben. Die vergangenen Reformen dürften unter anderem auf dem Wege einer größeren Konzessionsbereitschaft der Arbeitnehmer mit zu diesem Ergebnis beigetragen haben - in welchem Umfang, lässt sich zum gegenwärtigen Zeitpunkt jedoch noch nicht abschätzen. 


\subsection{ZWISCHENFAZIT UND VERBLEIBENDE HERAUS- FORDERUNGEN}

In den letzten Jahren konnten bei der Bekämpfung der eingangs genannten Probleme auf dem deutschen Arbeitsmarkt beträchtliche Fortschritte erzielt werden, die nicht bloß dem günstigen makroökonomischen Umfeld, sondern auch maßgeblich den umfassenden Reformen geschuldet sein dürften. Die Beschäftigung hat deutlich und stärker als in der Vergangenheit zugenommen, und dies gerade auch bei den wirtschaftspolitisch besonders ,wertvollen" Vollzeitstellen und der sozialversicherungspflichtigen Beschäftigung. Die Arbeitslosigkeit ist ganz erheblich zurückgegangen, und zwar nicht nur zyklisch, sondern auch in ihrem verfestigten Teil. Die Erwerbsbeteiligung Älterer hat merklich zugenommen. Und schließlich sind die aktive Arbeitsmarktpolitik und die sie umsetzenden Institutionen transparenter, günstiger und zielgenauer geworden.

Gleichwohl besteht eine Reihe von Problemen fort:

(1) Trotz des Rückgangs der Arbeitslosigkeit insgesamt sind die Unterbeschäftigung von Geringqualifizierten und der Anteil der Langzeitarbeitslosen weiterhin überdurchschnittlich hoch. Damit hängt zusammen, dass es erstens nicht gelungen ist, die Abgaben für einfache Tätigkeiten, die für viele dieser Arbeitslosen eine erste oder sogar auf absehbare Zeit die einzige Beschäftigungsmöglichkeit bieten, merklich zu senken. Zweitens weisen die Hinzuverdienstmöglichkeiten in der Grundsicherung für Arbeitsuchende den Konstruktionsfehler auf, dass sie die Aufnahme einer geringfügigen Beschäftigung sehr attraktiv gestalten, aber für den Übergang in eine niedrig entlohnte sozialversicherungspflichtige Voll- oder Teilzeittätigkeit eine hohe Schwelle setzen (SVR 2006a, Tz. 102ff.).

(2) Hinsichtlich des gesellschaftlichen und sozialpolitischen Umgangs mit nicht Existenz sichernden Löhnen fehlt es weiterhin an breiter Akzeptanz dafür, dass bestimmte Personen aufgrund geringer Produktivität und damit niedriger Löhne als Aufstocker längerfristig auf bedürftigkeitsabhängige staatliche Transfers angewiesen sein werden. Dieses Akzeptanzproblem verschärft sich noch, wenn die Betroffenen mit ihrem Arbeitseinkommen nicht nur sich selbst versorgen müssen und wenn, wie in den letzten Jahren, die Lohnspreizung in Deutschland vor allem in der unteren Hälfte der Lohnverteilung zugenommen hat (OECD 2009). In der öffentlichen Wahrnehmung dominiert allzu oft das vermeintliche Stigma des Transferbezugs statt der eigentlich gebotenen Anerkennung für den Eigenbeitrag zur Verringerung der Hilfebedürftigkeit, die $\$ 1$ Abs. 1 Nr. 1 SGB II ja als ein Ziel der Grundsicherung nennt. Mit dazu beigetragen haben dürfte nicht zuletzt die irreführende, mehr als unglückliche Bezeichnung „Arbeitslosengeld II“.

(3) Der zeitweise strukturell ausgeglichene Haushalt der Arbeitslosenversicherung ist durch eine zu weitgehende Senkung des Beitragssatzes inzwischen wieder unterfinanziert und belastet den Bundeshaushalt in erheblichem Umfang. Über den Zuschuss im laufenden Jahr hinaus ist zumindest in den beiden kommenden Jahren mit der Inanspruchnahme von Bundesdarlehen zu rechnen (BMF 2010, S. 51), die dann in den Folgejahren aus Überschüssen zurückzuzahlen sind, bevor die Bundesagentur daran gehen kann, neue Rücklagen aufzubauen.

(4) Die Segmentierung des Arbeitsmarktes wurde durch die arbeitsrechtliche Liberalisierung atypischer Beschäftigungsverhältnisse statt einer moderaten Liberalisierung in der Breite - Stichwort Kündigungsschutz - noch verstärkt (SVR 2008, Tz. 531ff.; kritisch dazu Pfarr et al. 2005). Dieser Punkt ist gerade mit Blick auf die mittelfristigen Arbeitsmarktwirkungen der Finanzkrise bedeutsam. Während die vergangenen Reformen auf jeden Fall die Anpassungsfähigkeit des Arbeitsmarktes bei zyklischen Schwankungen der Arbeitsnachfrage erhöht haben, bleibt abzuwarten, wie gut der Arbeitsmarkt auf einen künftigen Strukturanpassungsbedarf wegen eines niedrigeren Wachstums der Weltwirtschaft und auf wichtigen Absatzmärkten reagiert. Denn diese Anpassungen träfen zu einem überproportional hohen Anteil die Kernbelegschaft aus Qualifizierten und Hochqualifizierten, die von der Segmentierung des Arbeitsmarktes bislang durch größere Beschäftigungssicherheit eher noch profitiert hat.

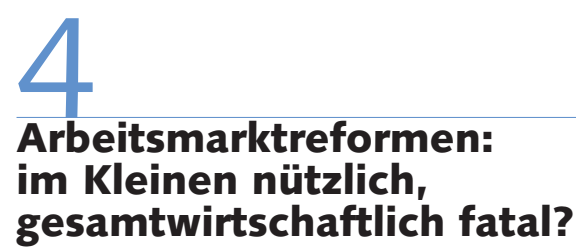

Ein wiederkehrender Streitpunkt in der Diskussion um Arbeitsmarktpolitik und Lohnentwicklung sind die gesamtwirtschaftlichen Rückwirkungen. Anschauliche Beispiele hierfür liefern die Jahresgutachten des SVR und die darin regelmäßig enthaltenen Minderheitsvoten zur Arbeitsmarktpolitik (z. B. SVR 2003, Tz. 659ff.; SVR 2005b, Tz. 322ff.). Diese Debatte wird erst recht mit Blick auf die Ausgestaltung und den Nutzen der jüngsten, weitreichenden Arbeitsmarktreformen geführt. Die mit den gesamtwirtschaftlichen Rückwirkungen argumentierende Kritik kann in etwa so gefasst werden: Zwar mag sich auf der mikroökonomischen Ebene die Funktionsfähigkeit des Arbeitsmarktes etwas verbessert haben, indem etwa Friktionen bei der Stellenbesetzung verringert und ein Teil der strukturellen Arbeitslosigkeit abgebaut wurden. Zugleich hatten die Reformen jedoch einen wesentlichen Anteil an der Schwäche der deutschen Binnenwirtschaft im vergangenen Aufschwung. Mehr noch, sie hätten über die Binnenschwäche und die Verbesserung der preislichen Wettbewerbsfähigkeit infolge geringer Lohnzuwächse mittelbar die Leistungsbilanzungleichgewichte im Euroraum und in der Weltwirtschaft verschärft (vgl. Sturn/van Treeck in diesem Heft). Träfe dieser Vorwurf zu, so würde er nicht nur den bereits beschriebenen Erfolg der Arbeitsmarktreformen relativieren. Er hätte auch beträchtliche Konsequenzen für die aktuelle wirtschaftspolitische Debatte über geeignete Maßnahmen zur Sicherstellung eines nachhaltigeren Wachstums der Weltwirtschaft und den Abbau der Divergenzen innerhalb des Euroraums. Denn in dem Maß, in dem die deutsche Volkswirtschaft und ihr auf Exportstärke ausgerichtetes „Geschäftsmodell“ diese Entwicklungen mit zu verantworten haben, scheint es legitim, von Deutschland einen aktiven fiskalpolitischen und „lohnpolitischen“ Beitrag zur Verringerung des Leistungsbilanzüberschusses einzufordern. Die Stichhaltigkeit solcher Einwände soll deswegen abschließend genauer ausgeleuchtet werden. 


\subsection{ARBEITSMARKTREFORMEN ALS URSACHE DER SCHWACHEN BINNENNACHFRAGE?}

Im vergangenen Aufschwung stützte sich der Anstieg der deutschen Wirtschaftsleistung rund zur Hälfte und damit in der Tat ungewöhnlich stark auf den Außenbeitrag. Für sich genommen haben die Reformen über einen verhaltenen Lohnanstieg und die zunehmende Lohnspreizung, soweit diese die Folge einer reformbedingt strukturell sinkenden Arbeitslosigkeit und sinkender Reservationslöhne sind, ${ }^{2}$ die Zunahme der verfügbaren Einkommen der Arbeitnehmer und damit die Binnennachfrage gedämpft.

Gegenzurechnen ist allerdings eine Reihe weiterer Effekte, die die Rolle der Arbeitsmarktreformen für die Binnenschwäche erheblich relativieren:

(1) Der durch die Reformen induzierte Beschäftigungsanstieg führt mit Blick auf das aggregierte verfügbare Einkommen zu einem Mengeneffekt, der dem schwächeren Anstieg der Pro-Kopf-Verdienste entgegenwirkt. Ähnlich wirken die im Zuge der sinkenden Arbeitslosigkeit gestiegene Beschäftigungssicherheit sowie die höhere Austrittswahrscheinlichkeit aus der Arbeitslosigkeit. Sie erhöhen zwar nicht das laufende verfügbare Einkommen, wohl aber den davon konsumierten Anteil, da die Notwendigkeit zu Vorsichtssparen für Phasen der Arbeitslosigkeit abnimmt.

(2) Anhaltend hohe Arbeitslosigkeit hat beträchtliche finanzielle und soziale Kosten, lässt gesamtwirtschaftliche Produktionsmöglichkeiten brachliegen und führt $\mathrm{zu}$ einer starken Beeinträchtigung des individuellen Wohlbefindens. Der Rückgang der strukturellen Arbeitslosigkeit bewirkt angesichts dessen einen spürbaren Wohlfahrtsgewinn, der nicht nur einmalig, sondern in jeder künftigen Periode erneut anfällt.

(3) Darüber hinaus gibt es weitere wichtige Ursachen für die verhaltene Binnennachfrage. Neben einer trotz des starken Anstiegs der Unternehmens- und Vermögenseinkommen niedrigen Investitionsquote zählt dazu insbesondere die ausgabenund einnahmeseitige Konsolidierung der öffentlichen Haushalte. Dadurch gelang die nicht bloß zyklisch bedingte, sondern strukturelle Rückführung der öffentlichen
Defizite, was die gerade in der Krise essenzielle Wiedergewinnung der finanzpolitischen Handlungsfähigkeit ermöglicht hat, nachdem Deutschland in den Jahren zuvor wiederholt gegen die 3-\%-Grenze des Stabilitäts- und Wachstumspaktes verstoßen und so, nebenbei bemerkt, ebenfalls zu dessen nachlassender Bindungswirkung beigetragen hatte.

(4) Mit Blick auf die künftige Entwicklung ist wichtig, dass der dämpfende Beitrag von Lohnmoderation kein dauerhaftes Phänomen, sondern eine Anpassungsreaktion an eine dauerhaft niedrigere Arbeitslosenquote ist. ${ }^{3}$ Mittelfristig ist bei Beibehaltung der Reformen auch mit einem wieder stärkeren Wachstum der Binnennachfrage zu rechnen. Ein Indiz hierfür ist der Anstieg der nominalen verfügbaren Einkommen im Jahr 2008. Zwar wurde der Einkommenszuwachs durch den rapiden Anstieg der Rohstoffpreise wieder aufgezehrt; gleichwohl wirkte die anziehende Binnenkonjunktur dann noch in die Krise hinein stabilisierend. Dies zeigte sich beispielsweise am - trotz des starken Einbruchs der Wirtschaftsleistung - robusten Konsum und der beschriebenen gegenläufigen Beschäftigungsentwicklung von exportabhängigeren und eher binnenwirtschaftlich ausgerichteten Sektoren.

\subsection{BEITRAG ZU DIVERGENZEN IM EURORAUM UND INTERNATIONALEN UNGLEICHGEWICHTEN?}

Bereits vor Ausbruch der Finanzkrise und verstärkt seit dem letzten Jahr sah sich Deutschland auf internationaler Ebene mit Vorhaltungen konfrontiert, seine Lohnzurückhaltung und die damit verbundene Exportstärke seien für die zunehmenden Divergenzen innerhalb des Euroraums sowie für die persistenten internationalen (Leistungsbilanz-)Unterschiede mit verantwortlich. Daraus wird die Forderung abgeleitet, Deutschland müsse als Beitrag zum Abbau dieser „Ungleichgewichte“ und damit für ein nachhaltigeres Wachstum der Weltwirtschaft eine expansivere Fiskal- und Lohnpolitik betreiben (vgl. u. a. Sturn/van Treeck in diesem Heft). Diese Argumentation und die Schlussfolgerungen sind jedoch aus folgenden Gründen abzulehnen:

(1) Exportstärke darf nicht mit Leistungsbilanzüberschüssen gleichgesetzt werden
- hohe Exporte können durchaus auch mit Leistungsbilanzdefiziten einhergehen. Entscheidend für den Leistungsbilanzüberschuss ist das Verhältnis von Exporten und Importen.

(2) Die deutschen Exportüberschüsse sind keineswegs vor allem auf einen Anstieg der preislichen Wettbewerbsfähigkeit infolge Lohnmoderation zurückzuführen. Wesentlich wichtiger für die vergangenen Exporterfolge war ein starkes Wachstum der Weltwirtschaft, das zudem in seiner regionalen und qualitativen Zusammensetzung (etwa Investitionsgüter und langlebige Konsumgüter wie Automobile) das Gütersortiment der deutschen Wirtschaft begünstigt hat.

(3) Es ist unzutreffend, im Zusammenhang mit der deutschen Exportstärke von einem „Geschäftsmodell“ oder einer moderaten „Lohnpolitik“ zu sprechen. Die Exporterfolge sind das Ergebnis der Leistungen der Unternehmen und ihrer Beschäftigten, und anders als in manchen Schwellenländern gab und gibt es in Deutschland keine wirtschaftspolitische Strategie der Exportförderung, etwa durch Wechselkursinterventionen oder eine aktive Industriepolitik. Die Löhne wiederum werden in Deutschland nicht wirtschaftspolitisch gesetzt, sondern sind das Ergebnis individueller oder kollektiver Lohnverhandlungen zwischen Arbeitgebern und Arbeitnehmern. Einen merklichen und vor allem vorsätzlichen Einfluss der Politik auf die Lohnsetzung, wie ihn der Begriff „Lohnpolitik“ suggeriert, gibt es nur am unteren Ende der Lohnskala durch verbindliche Lohnuntergrenzen über Allgemeinverbindlicherklärungen und branchenspezifische Mindestlöhne. Diese sind indessen für die Exporterfolge irrelevant und tragen je nach Ausgestaltung eher zur Schwäche der Binnennachfrage bei, wenn

2 Andere lohndämpfende Faktoren, die nicht den Arbeitsmarktreformen zuzurechnen wären, sind beispielsweise zunehmender Wettbewerb auf den Märkten für Güter und Dienstleistungen durch den Aufholprozess der Schwellenländer oder technischen Fortschritt, der die Austauschbarkeit von Gütern und Dienstleistungen und damit ebenfalls die Wettbewerbsintensität für die jeweiligen Anbieter erhöht.

3 Analoges gilt für die nachfragedämpfende Wirkung der Haushaltskonsolidierung und den damit verbundenen Übergang zu einer niedrigeren staatlichen Neuverschuldung. 
sie Tätigkeiten und Sektoren, die netto zur Erhöhung der inländischen Absorption führen dürften, in ihrer Entwicklung behindern. Die Mindestlöhne im Postgewerbe, die, obgleich nur zeitweise in Kraft, die Verdrängung von Wettbewerbern zumindest begünstigt haben, sind dafür ein anschauliches Beispiel.

(4) Selbst wenn man unterstellte, die Arbeitsmarktreformen hätten mittelbar über niedrige Lohnsteigerungen die deutsche Binnenkonjunktur geschwächt, die Exporterfolge maßgeblich getrieben und so in der Summe zu den Leistungsbilanzüberschüssen beigetragen, wäre es dennoch verfehlt, Deutschland daher (und wegen der mit den Leistungsbilanzüberschüssen einhergehenden Kapitalexporte) als mitverantwortlich für die Probleme der Peripherieländer innerhalb des Euroraums oder gar die globalen Ungleichgewichte zu machen. Die hohen Leistungsbilanzdefizite in einer Reihe von Mitgliedsländern der Europäischen Währungsunion (EWU) sind die Folge einer dort überzogenen, auf die Dauer nicht durchzuhaltenden Binnendynamik (Bundesbank 2010b). Die betreffenden Länder haben den mit dem Eintritt in die EWU verbundenen Zinsvorteil sowie den Wegfall des Wechselkursrisikos nicht genutzt, um ihre Wirtschaft auf einen dauerhaft höheren und steileren Wachstumspfad zu bringen. Vielmehr haben sie über eine zunehmende Verschuldung den privaten und staatlichen Konsum sowie Investitionen mit zweifelhaften Ertragsaussichten, insbesondere im Immobilienbereich, angeschoben. Dieses „Wachstumsmodell" war in der Tat auf beträchtliche Kapitalimporte aus dem Ausland angewiesen. Wo auch immer die Kapitalgeber saßen, es ist jedenfalls ein mehr als befremdlicher, letztlich die Empfängerländer bevormundender Vorwurf, wenn die Kapitalgeber und ihre aus guten Gründen neu ausgerichtete Arbeitsmarktpolitik für die mittels der Kapitalzuflüsse finanzierten Fehlentwicklungen verantwortlich gemacht werden. Es wäre Aufgabe der Wirtschaftspolitik in den Defizitländern gewesen, eine aus dem Ruder laufende Binnendynamik zu dämpfen. Mögliche Ansatzpunkte dafür wären

- eine restriktivere Finanzpolitik: sei es durch Abgabenerhöhungen, die Verkleinerung eines überdimensionierten öffentlichen Sektors oder Leistungsrücknahmen im Rentensystem, um dessen Tragfähigkeit wiederherzustellen;

- die Liberalisierung des Arbeitsmarktes, welche, wie in Deutschland, die Erwerbsbeteiligung erhöht und die häufig trotz starken BIP-Wachstums immer noch hohe Arbeitslosigkeit und damit den Lohndruck verringert hätte;

- oder eine strengere Bankenaufsicht zur Dämpfung der Kreditvergabe im Immobilienbereich.

In ähnlicher Weise hätten Strukturreformen zur Verbesserung der Angebotsbedingungen, etwa im Schulsystem oder bei den durch Verwaltungshandeln bestimmten Standortbedingungen für Unternehmen (Bürokratieabbau, Effizienz des Steuersystems) das Produktivitätswachstum erhöht und den Verlust an Wettbewerbsfähigkeit zumindest gedämpft. Im Ergebnis wäre die inländische Absorption zwar niedriger gewesen, die Verschuldung von Unternehmen, Haushalten und der öffentlichen Hand im Ausland hätte aber bei Weitem nicht so stark zugenommen, dass mittlerweile sogar die Kreditwürdigkeit von Staaten infrage gestellt wird. Im Gegenzug wäre das deutsche Exportvolumen marktgetrieben und ganz ohne makroökonomische Feinsteuerung geringer ausgefallen. Die Politik in den betreffenden Ländern hat bekanntermaßen anders entschieden und ist nun gezwungen, alle diese Maßnahmen in größerer Eile und in einem deutlich ungünstigeren wirtschaftlichen Umfeld nachzuholen. Aus diesen wirtschaftspolitischen Versäumnissen der Defizitländer nun aber eine Verantwortung der deutschen Volkswirtschaft und der dortigen Arbeitsmarktpolitik zu konstruieren, stellt die Kausalzusammenhänge auf den Kopf und steht einer ursachengerechten Problemlösung im Weg. Künftig wird die - aufgrund der nunmehr unabwendbaren Anpassungsprozesse - gedämpfte Binnendynamik in den bisherigen Defizitländern auch die deutschen Exportvolumina senken und in Deutschland mehr Kapital und Arbeitskräfte in stärker binnenwirtschaftlich ausgerichtete Wirtschaftszweige fließen lassen. Diesen Anpassungsprozess gilt es gegebenenfalls abzufedern, aber nicht durch die wie auch immer geartete Forcierung höherer, nicht marktgerechter Lohnabschlüsse (etwa über Appelle an die Tarifvertragsparteien oder den Versuch einer Lohnführerschaft des öffentlichen Dienstes) noch zu beschleunigen. Das
Gros der Anpassung zum Abbau der Divergenzen innerhalb des Euroraums und der Leistungsbilanzungleichgewichte in der Weltwirtschaft - zu denen der Euroraum als Ganzes bekanntermaßen nicht beigetragen hat - muss in den bisherigen Defizitländern durch eine Korrektur der dortigen Rahmenbedingungen erfolgen. Die Arbeitsmarktreformen in Deutschland sind dafür keine Warnung, sondern ein ermutigendes Beispiel.

In der Summe bleibt daher festzuhalten, dass die aus gesamtwirtschaftlicher Sicht vorgebrachten Argumente gegen den Nutzen der Arbeitsmarktreformen nicht verfangen.

\section{Fazit: Die Richtung stimmt}

Bei der Bewältigung der eingangs genannten wirtschaftspolitischen Probleme konnten durch ein ganzes Paket an Maßnahmen beträchtliche Fortschritte erzielt werden. Der deutsche Arbeitsmarkt und mit ihm die gesamte Volkswirtschaft sind dank dieser Reformen in deutlich besserer Verfassung als zuvor. Für die Zukunft kommt es daher auf dreierlei an: erstens, das unter beträchtlichem finanziellen und politischen Kosten Erreichte nicht zu verspielen, etwa bei der Verkürzung der Bezugsdauer des Arbeitslosengeldes und der primär am Vermittlungserfolg ausgerichteten Auswahl arbeitsmarktpolitischer Instrumente; zweitens, Nachjustierungen bei erkannten Schwächen vorzunehmen, so bei den zu stark auf den Partizipationsgesichtspunkt statt die Aufnahme einer sozialversicherungspflichtigen Beschäftigung zielenden Hinzuverdienstmöglichkeiten im SGB II oder dem zu niedrigen Beitragssatz zur Arbeitslosenversicherung; und drittens gilt es, sich weiterhin mit fortbestehenden Problemen zu befassen, insbesondere mit der weiter unbefriedigenden Arbeitsmarktlage Geringqualifizierter und dem noch hohen Anteil der Langzeitarbeitslosen. Um die Durchlässigkeit des Arbeitsmarktes für alle Erwerbspersonen zu erhöhen, sollte eine schrittweise, den Bestandsschutz sichernde Lockerung des Kündigungsschutzes in der Breite erwogen werden. Im Gegenzug könnte dann die Segmentierung des Arbeitsmarktes wieder verringert werden, vor allem durch die 
Beseitigung von Ausnahmetatbeständen bei der sachgrundlosen Befristung und der geringfügigen Beschäftigung (SVR 2008, Tz. 531ff.). Klar ist aber auch, dass eine nachhaltige Erhöhung der Beschäftigung gerade bei diesen beiden Problemgruppen nicht allein mit arbeitsmarktpolitischen Instrumenten gelingen kann und wird, sondern komplementäre sozial- und bildungspolitische Reformen unverzichtbar sind.

\section{LITERATUR}

Arlt, A./Dietz, M./Walwei, U. (2009): Nicht alles ist Konjunktur Besserung für Ältere am Arbeitsmarkt, IAB-Kurzbericht 16, Nürnberg Bernhard, S./Hohmeyer, K./Jozwiak, E./Koch, S./Kruppe, T./Stephan, G./Wolff, J. (2009): Aktive Arbeitsmarktpolitik in Deutschland und ihre Wirkungen; in: Möller, J./Walwei, U.: Handbuch Arbeitsmarkt 2009, hrsg. vom Institut für Arbeitsmarkt- und Berufsforschung, Nürnberg Bundesagentur für Arbeit (BA) (2010): Arbeitsmarkt 2009, Amtliche Nachrichten der Bundesagentur für Arbeit 2, hrsg. von der Bundesagentur für Arbeit, Nürnberg

Bundesministerium der Finanzen (BMF) (2010): Monatsbericht Juli 2010, Berlin

Caliendo, M. (2009): Income Support Systems, Labor Market Policies and Labor Supply: The German Experience, IZA Discussion Paper 4665, Bonn

Deutsche Bundesbank (Bundesbank) (2009): Perspektiven der deutschen Wirtschaft - Gesamtwirtschaftliche Vorausschätzungen 2010 und 2011, in: Deutsche Bundesbank (Hrsg.): Monatsbericht Dezember, Frankfurt am Main

Deutsche Bundesbank (Bundesbank) (2010a): Perspektiven der deutschen Wirtschaft - Gesamtwirtschaftliche Vorausschätzungen 2010 und 2011, in: Deutsche Bundesbank (Hrsg.): Monatsbericht Juni, Frankfurt am Main, S. 15-28

Deutsche Bundesbank (Bundesbank) (2010b): Zur Problematik makroökonomischer Ungleichgewichte im Euro-Raum, in: Deutsche Bundesbank (Hrsg.): Monatsbericht Juli, Frankfurt am Main, S. 17-40

Dlugosz, S./Stephan, G./Wilke, R. (2009): Deutliche Effekte auf die Eintritte in Arbeitslosigkeit - Verkürzte Bezugsdauer für Arbeitslosengeld, IAB-Kurzbericht 30, Nürnberg

Hohendanner, C. (2010): Unsichere Zeiten, unsichere Verträge? Befristete Arbeitsverträge zwischen Auf- und Abschwung, IAB-Kurzbericht 14, Nürnberg

Horn, G./Logeay, C./Stapf, D. (2007): Viel Lärm um nichts? Arbeitsmarktreformen zeigen im Aufschwung bisher kaum Wirkung, IMK Report 20

Horn, G./Logeay, C./Zwiener, R. (2008): Wer profitierte vom Aufschwung?, IMK Report 27

Internationaler Währungsfonds (IWF) (2010): World Economic Outlook October 2010. Recovery, Risk and Rebalancing, Washington D.C. Jacobi, L./Kluve, J. (2007): Before and after the Hartz reforms: The performance of active labour market policy in Germany, in: Zeitschrift für ArbeitsmarktForschung 1, S. 45-64
Konle-Seidl, R./Eichhorst, W./Grienberger-Zingerle, M. (2007): Activation Policies in Germany - From Status Protection to Basic Income Support, IAB Discussion Paper 6, Nürnberg

Möller, J./Walwei, U./Koch, S./Kupka, P./Steinke, J. (2009): Der Arbeitsmarkt hat profitiert - Fünf Jahre SGB III: Eine IAB-Bilanz, IABKurzbericht 29, Nürnberg Organisation für wirtschaftliche Zusammenarbeit und Entwicklung (OECD) (2009): OECD Employment Outlook 2009 (Statistical Annex), Paris

Pfarr, H./Ullmann, K./Bradtke, M./Schneider, J./Kimmich, M./Bothfeld, S. (2005): Der Kündigungsschutz zwischen Wahrnehmung und Wirklichkeit. Betriebliche Erfahrungen mit der Beendigung von Arbeitsverhältnissen, München/Mehring

Sachverständigenrat zur Begutachtung der gesamtwirtschaftlichen Entwicklung (SVR) (2002): Zwanzig Punkte für Beschäftigung und Wachstum, Jahresgutachten 2002/2003, Wiesbaden Sachverständigenrat zur Begutachtung der gesamtwirtschaftlichen Entwicklung (SVR) (2003): Staatsfinanzen konsolidieren - Steuersystem reformieren, Jahresgutachten 2003/2004, Wiesbaden

Sachverständigenrat zur Begutachtung der gesamtwirtschaftlichen Entwicklung (SVR) (2005): Die Chance nutzen - Reformen mutig voranbringen, Jahresgutachten 2005/2006, Wiesbaden Sachverständigenrat zur Begutachtung der gesamtwirtschaftlichen Entwicklung (SVR) (2006a): Arbeitslosengeld II reformieren: Ein zielgerichtetes Kombilohnmodell, Expertise, Wiesbaden

Sachverständigenrat zur Begutachtung der gesamtwirtschaftlichen Entwicklung (SVR) (2006b): Widerstreitende Interessen - ungenutzte Chancen, Jahresgutachten 2006/2007, Wiesbaden

Sachverständigenrat zur Begutachtung der gesamtwirtschaftlichen Entwicklung (SVR) (2007): Das Erreichte nicht verspielen, Jahresgutachten 2007/2008, Wiesbaden

Sachverständigenrat zur Begutachtung der gesamtwirtschaftlichen Entwicklung (SVR) (2008): Die Finanzkrise meistern - Wachstumskräfte stärken, Jahresgutachten 2008/2009, Wiesbaden

Sachverständigenrat zur Begutachtung der gesamtwirtschaftlichen Entwicklung (SVR) (2009): Die Zukunft nicht aufs Spiel setzen, Jahresgutachten 2009/2010, Wiesbaden 